\title{
TIBIALIS POSTERIOR TRANSFER IN CONGENITAL CLUB FOOT
}

\author{
Martin Singer, Cape Town, South Africa
}

In a review of the results of tibialis anterior transfer in congenital club foot it was found that fifty-two relapses occurred in a series of seventy-six tendon transfers (Singer and Fripp 1958). In addition certain undesirable sequelae resulted from the operation: 1) a dorsiflexed (cocked-up) hallux and dropped first metatarsal head in fifty-seven instances; and 2) disturbed muscle balance, the transposed muscle producing excessive pronation of the forefoot in eleven patients and excessive valgus of the hindfoot in three. The operation was based on the premise that in the presence of relative peroneal insufficiency the tibialis anterior muscle was the most powerful deforming factor causing relapse.

In all of the fifty-two relapses the principal component, and usually the only one, was the equinus deformity. The results suggested that the basic principle of the operation was incorrect. because the tibialis anterior is an important dorsiflexor of the foot as well as an invertor. In transferring it one might encourage the recurrence of equinus deformity because transfer always weakens a muscle. This tendency is aggravated by the power of the tibialis posterior muscle. The influence of this muscle is seen during the operation of soft-tissue correction (Brockman 1930), when invariably it must be lengthened to allow correction of the deformity. The power of the tibialis posterior was also demonstrated by Gunn and Molesworth (1957), who found it an effective dorsiflexor when transferred to the dorsum of the foot for lateral popliteal nerve paralysis in leprosy.

It was apparent that tibialis anterior transfer had an extremely limited place in the treatment of the difficult or relapsed club foot. An operative trial of transferring the tibialis posterior muscle through the interosseous membrane to the dorso-lateral aspect of the foot was undertaken. In this way a major deforming force would be removed and the tibialis posterior acting from its new insertion, although weakened, would supplement the relatively weak peroneal muscles and at the same time the dorsiflexor power of the tibialis anterior would be unimpaired. The retention of the tibialis anterior in its normal position might also prevent the development of the cocked-up hallux and a depressed first metatarsal head. This concept was supported in an editorial by Barr (1958).

\section{CLINICAL MATERIAL}

The patients in this series constitute the "difficult" group of club feet. They all had some residual deformity when treatment started and this was due to relapse of a previously corrected deformity or to incomplete initial correction or to lack of previous treatment. Eleven of the seventeen children were non-European. A total of twenty-eight feet in seventeen patients were corrected before operation by the Kite method (Fripp and Singer 1953). The ages of the patients varied from two and a half to eight and a half years at the time of the operation.

\section{TECHNIQUE OF OPERATION}

The tibialis posterior tendon is exposed through a curved incision behind the medial malleolus. The incision starts an inch above the tip of the malleolus and extends downwards as far as the tuberosity of the navicular bone. In every instance the tibialis posterior tendon was thicker than normal in this region, and in some patients the tendon had already split into two under cover of the malleolus. After detachment of the tendon as far distally as possible a strong stay suture is inserted into the cut end. 
A second incision, two and a half to three inches long, is made at the junction of the middle and distal thirds of the tibia. The incision skirts the crest of the tibia on its lateral aspect. Through this incision, and extraperiosteally, the interosseous membrane is exposed and an

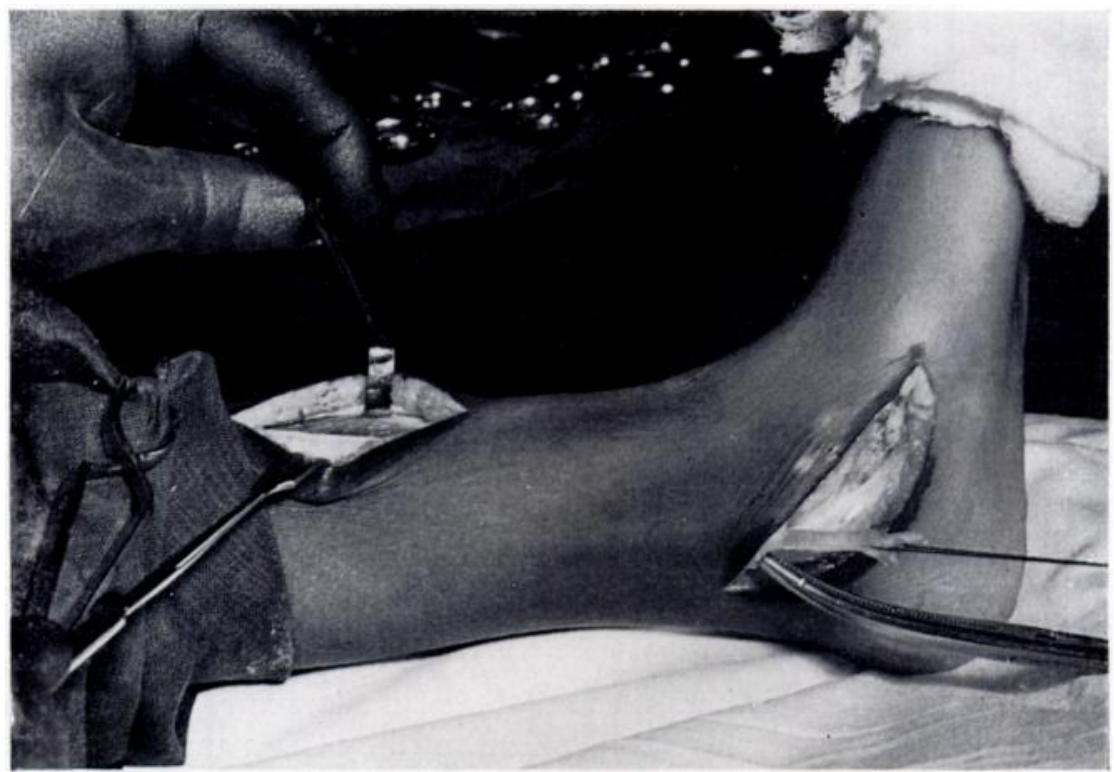

Fig. 1

Roberts' forceps passed proximally along the course of the tibialis posterior tendon hugging the tibia closely.

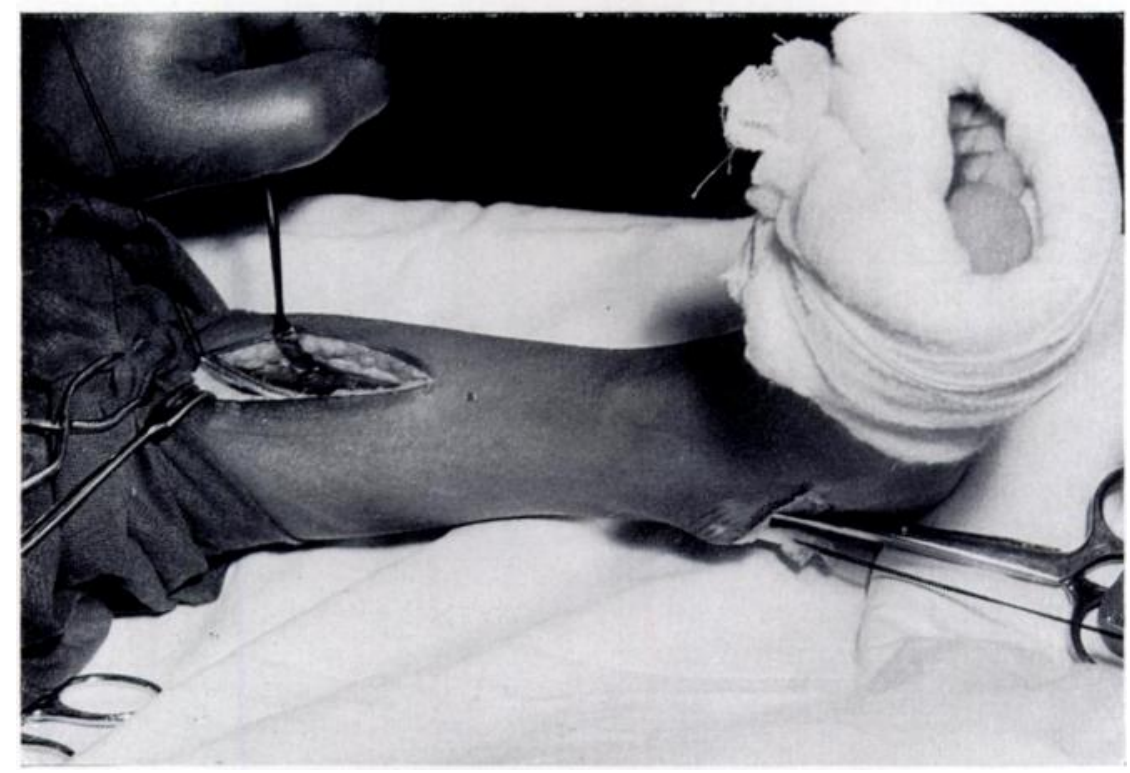

Fig. 2

The nose of the Roberts' forceps has been passed through the opening in the interosseous membrane and has grasped a double strand of strong thread.

opening the same length as the incision is made in it. A Roberts' forceps is introduced into the first incision and passed proximally, using the tendon of the tibialis posterior as a guide (Fig. 1). Hugging the tibia closely the forceps is pushed upwards through the aperture in 
the interosseous membrane and a double strand of strong suture material is withdrawn into the first incision (Fig. 2). After tying this to the stay suture it is easy to withdraw the tibialis posterior through the interosseous membrane. This is much easier and less traumatic than trying to introduce a forceps from above downwards.

The first incision is closed and a third incision made over the dorsolateral aspect of the foot. The tendon is drawn down subcutaneously into the third incision (Fig. 3). Before closing the second incision care must be taken to ensure that the part of the tibialis posterior muscle belly protruding through the opening in the interosseous membrane is not impinging on the sharp inelastic distal border. When necessary the gap in the interosseous membrane is enlarged distally to allow the transplanted tendon to glide freely.

In only one-third of the patients was it possible to secure the tendon to bone-either the lateral or the middle cuneiform-because the tendon was too short for safe anchorage. In the remainder the tendon was attached to the peroneus tertius tendon if this was present or to the lateral two tendons of extensor digitorum longus. This was done by the usual "buttonhole" method.

After operation the transplant is protected for six weeks in a below-knee

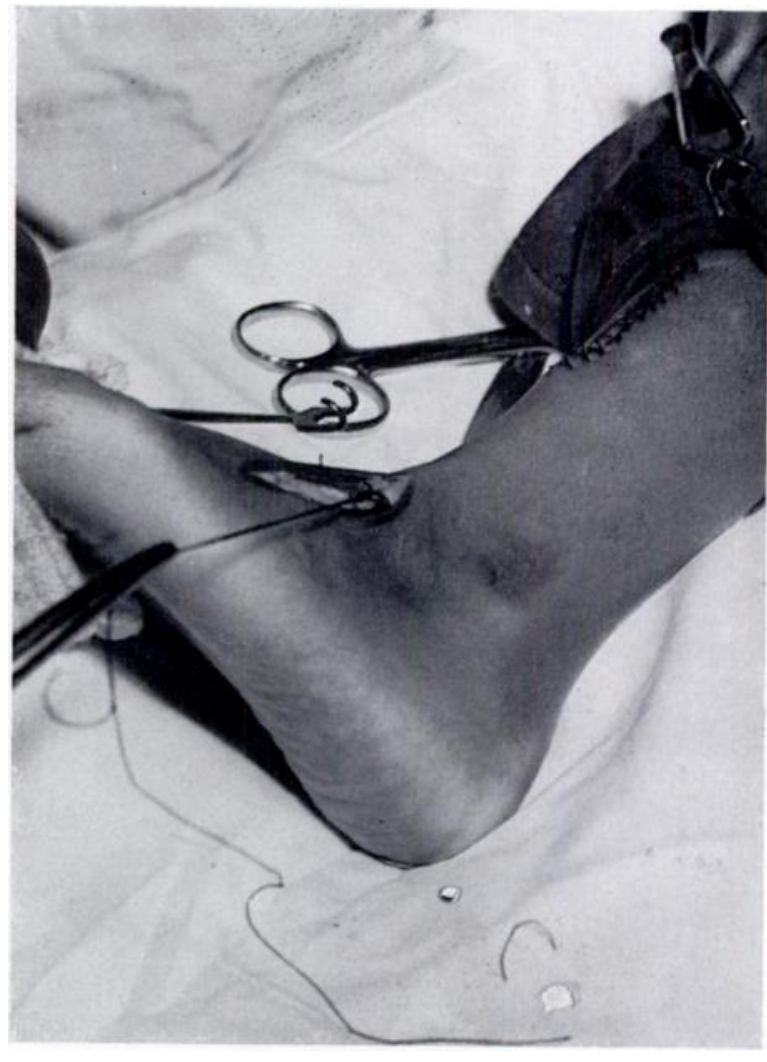

FIG. 3

The tibialis posterior tendon has been brought down to the dorso-lateral aspect of the foot for reinsertion. padded plaster with the foot in the over-corrected position. When the plaster is removed normal lace-up boots or shoes are worn. A strong plaster night splint is used for six months.

The view of Gunn and Molesworth (1957) that muscle re-education is easy after tibialis posterior transfer was confirmed in this series. No specialised retraining programme was adopted and no physiotherapists were employed for re-educative exercises. Many of the patients had active control of the transplant when the skin sutures were removed.

\section{RESULTS}

The criteria used to assess the results of tibialis anterior transfer (Singer and Fripp 1958) were again used in this series. A relapse was defined as the return of a foot to a state in which it was not plantigrade and either the adduction, inversion or equinus components of the deformity were not corrected to the neutral position. The assessment included the test for occult equinus (Singer and Fripp 1958).

The follow-up period varied from one year to three years. In twenty-seven of the twenty-eight feet operated on there was no relapse (Figs. 4 and 5). The shoes or boots wore evenly on soles and heels and no undesirable sequelae were observed except for a mild or moderate valgus of the hindfoot in four instances. This was possibly due to over-correction 

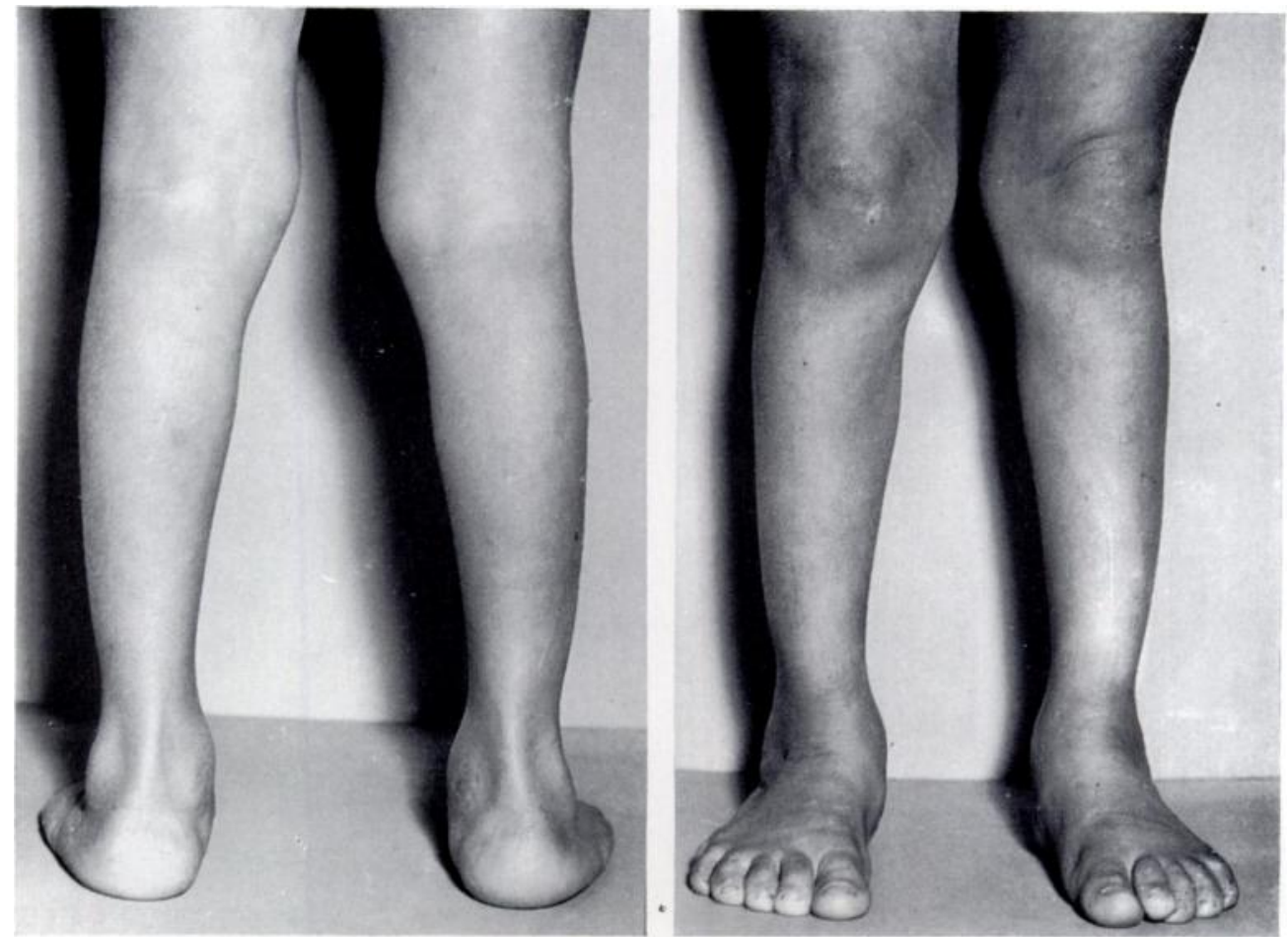

Fig. 4

Bilateral congenital club feet twenty-one months aftar tibialis posterior transfer.

before operation. The amount of valgus has remained unchanged until the present. There was a relapse in one child due to the disinsertion of the tendon at its new attachment. The disinsertion may have been due partly to the fact that this patient was discharged from hospital after two weeks and only reappeared six months later walking barefooted. It was interesting

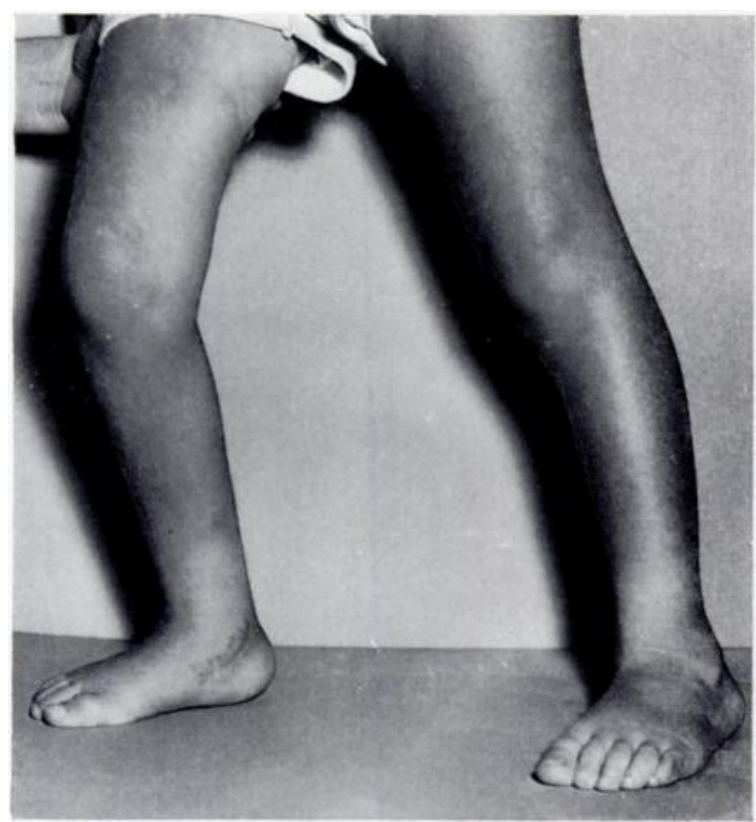

FiG. 5

Negative test for occult equinus. that the relapse occurred in the direction of the adduction rather than the equinus component of the deformity.

The operation of soft-tissue correction was performed in five feet, which are not included in this series, during the phase of varus wedging to correct the forefoot adduction. When the tibialis posterior tendons were exposed later all five were found unsuitable for transfer because they were thin, atrophic and adherent to the surrounding tissues.

Great care was taken to ensure that all three components of the deformity were completely corrected before the operation. This cardinal rule of tendon transference was relaxed in one instance and relapse occurred three months after the operation. This patient is not included in the series because the treatment began after this review had been completed. 


\section{SUMMARY}

1. The tibialis posterior tendon was transferred in twenty-eight congenital club feet to maintain the correction obtained by serial wedge plasters.

2. There has been no relapse in twenty-seven of the twenty-eight feet in the period under review-namely, one to three years from operation.

3. The technique of the operation is described.

4. It appears that this operation should not be attempted when a soft-tissue correction has been done previously.

Since the completion of this paper Fried (1959) has reported thirteen patients on whom this operation has been performed. Twelve of the patients had results classified as excellent and satisfactory with a follow-up period of four to five years.

I have much pleasure in recording my thanks to my brother, Mr Alec Singer, for his constant encouragement and helpful criticism.

\section{REFERENCES}

BARR, J. S. (1958): Tendon Transplantation. Journal of Bone and Joint Surgery, 40-B, 166.

Brockman, E. P. (1930): Congenital Club-foot. Bristol: John Wright \& Sons Ltd.

FrIED, A. (1959): Recurrent Congenital Club-Foot. Journal of Bone and Joint Surgery, 41-A, 243.

Fripp, A. T., and Singer, M. (1953): The Kite Treatment of Congenital Talipes Equino-varus. Postgraduate Medical Journal, 29, 391.

Gunn, D. R., and Molesworth, B. D. (1957): The Use of Tibialis Posterior as a Dorsiflexor. Journal of Bone and Joint Surgery, 39-B, 674.

Singer, M., and FrIPP, A. T. (1958): Tibialis Anterior Transfer in Congenital Club Foot. Journal of Bone and Joint Surgery, 40-B, 252. 